\title{
A Hybrid Approach for Movie Recommendation based on User Behaviour
}

\author{
Minal V. Jamnekar ${ }^{1}$, Prof. Sneha U. Bohra ${ }^{2}$
}

${ }^{1}$ M. Tech Student, Department of Computer Science Engineering, G. H. Raisoni University, Amaravti, Maharashtra, India

2Maharashtra, India Department of Computer Science Engineering, G. H. Raisoni University, Amravati, Maharashtra, India

\begin{abstract}
Article Info

Volume 8, Issue 3

Page Number : 543-550

Publication Issue

May-June-2021

Article History

Accepted : 03 June 2021

Published : 08 June 2021

The propose framework carrying out a hybrid approach for the movie suggestion dependent on user behavior that upgrades the properties of the previous framework with a more up-to-date and more productive methodology that lessens the framework run time and decides thing relations with more prominent precision. To develop a hybrid model fit for making a decent proposal dependent on metadata about the movie and the behavior of the user. To assess the proposed framework on the boundary of RMSE.

Keywords : Movie Recommendation, User Behavior, Content Based Filtering Recommendation, Collaborative Filtering Recommendation, User Based Recommendation, Item Based Recommendation, Target User, Similar Users, Rating
\end{abstract}

\section{INTRODUCTION}

Recommender frameworks are PC-based astute procedures to manage the issue of data and item overburden. They dissect examples of user interest in items and things to give customized proposal benefits that match the user's inclination, interest, and decision. Recommender frameworks establish a particular kind of data sifting that endeavours to introduce things as per the interests communicated by a user. Recommender frameworks are utilized to make suggestions about data, items, or services for users. We propose a hybrid recommender framework for movie recommendations dependent on user interaction and behaviour. The recommender framework acknowledges user demands, prescribes $\mathrm{N}$ things to the user and records user interest, decision, preference, and choice.

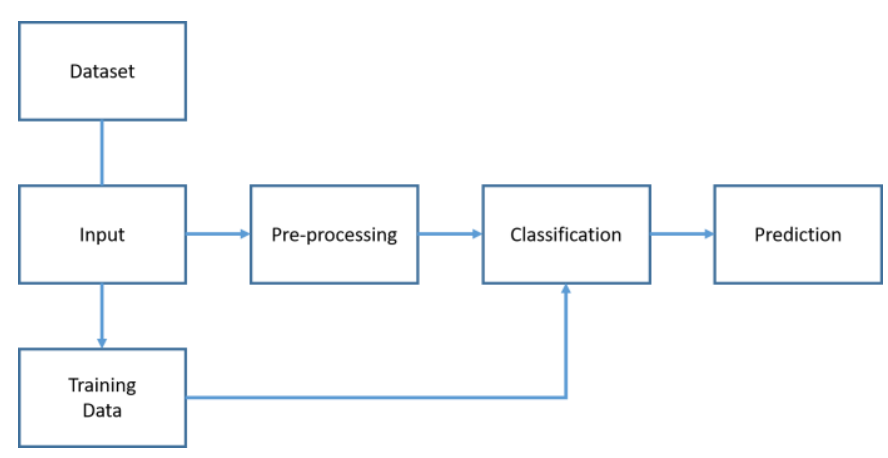

Fig 1: Block Diagram

Copyright: () the author(s), publisher and licensee Technoscience Academy. This is an open-access article distributed under the terms of the Creative Commons Attribution Non-Commercial License, which permits unrestricted non-commercial use, distribution, and reproduction in any medium, provided the original work is properly cited 
There are most common types of recommendation systems are as follows:

a) Content-based Filtering Recommendation and

b) Collaborative Filtering Recommendation

c) Hybrid Filtering Recommendation

a) Content-based Filtering Recommendation

This filtering recommends things to user dependent on his past experience. For instance, assuming a user loves just activity movie, the framework predicts his solitary activity movies like it which he has profoundly appraised. Thing/content for the most part indicated by watchwords. It just spotlights a solitary user's thoughts, contemplations and gives expectations dependent on his advantage.

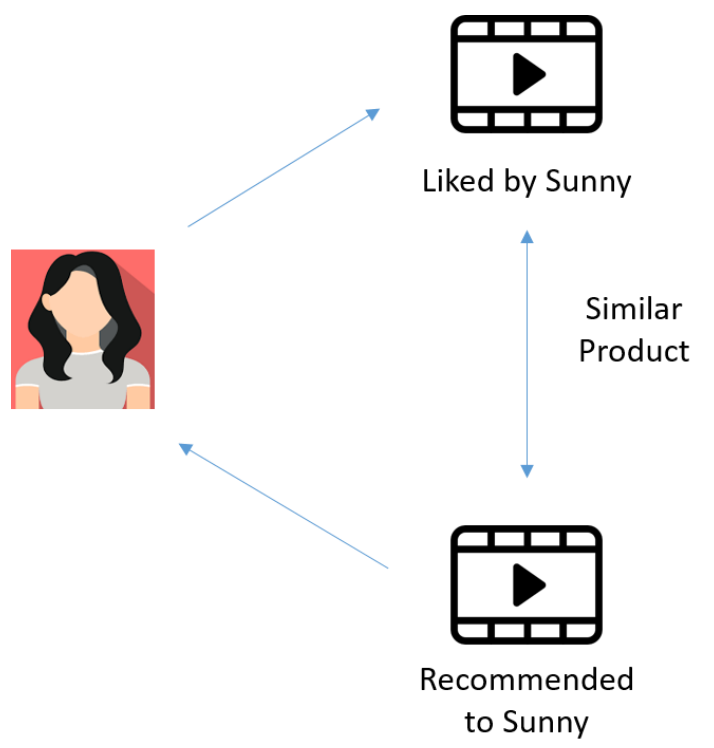

Fig 2: Content-based Filtering Recommendation

\section{b) Collaborative Filtering Recommendation}

Collaborative Filtering procedures make suggestions for a user dependent on ratings and conduct information of numerous users. Assuming two users have both preferred certain regular things, the things that one user has loved that the other user. More users, more ratings: better outcomes.

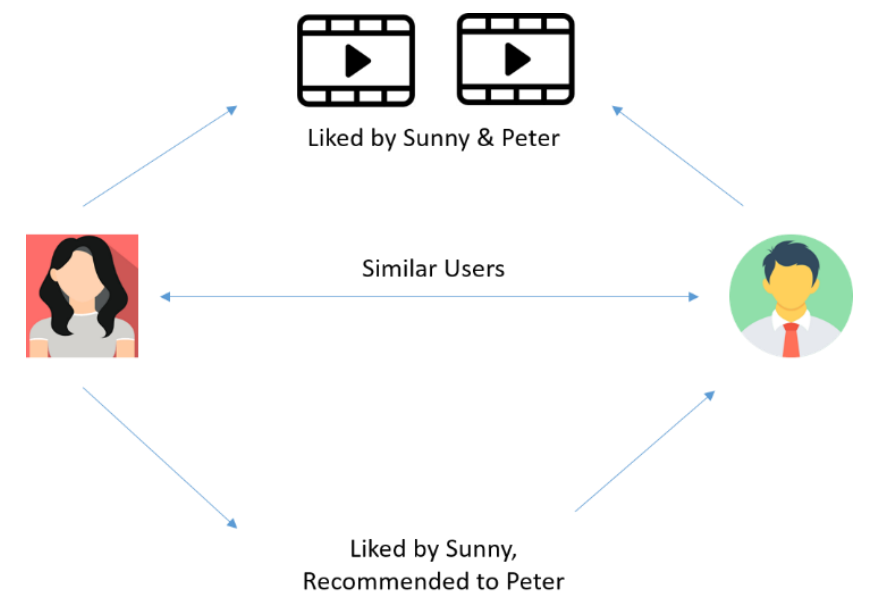

Fig 3: Collaborative Filtering Recommendation

c) Hybrid Filtering Recommendation

It is a mix of the two strategies for example Shared collaborative filtering recommendation framework and content-based filtering recommendation framework. This hybrid filtering accepts ratings of the movie as the contribution from the users and afterward applies the collaborative filtering and content-based filtering and produces a suggestion list.

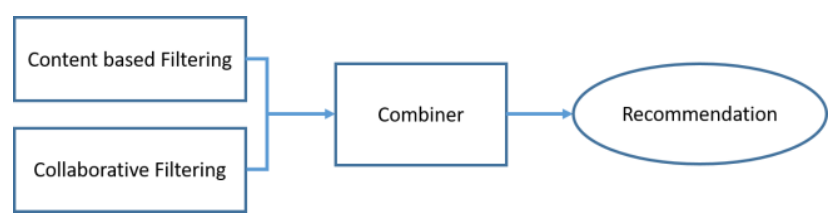

Fig 4: Hybrid Filtering Recommendation

\section{Problem Statement}

The propose framework carrying out a crossover suggestion framework for movie proposals dependent on user decision, choice, interest, preferences, and behaviour that develop the properties of the past framework with a novel methodology and extra fit methodology that abatement the framework run-time and decides thing relationship with immense flawlessness.

\section{Literature Survey}

Pooja Khalokar, et al. [1] Portray a model is proposed to tackle the movement suggestion issue by dealing with the fascination subject, vacationer inclination, 
and feeling of the attractions. It will take constant information at that point examine it and utilize the information for ongoing applications to suggest the vacationer's real right intrigued information. The proposed Sentiment information model can be additionally applied for the voyaging suggestion applications, like fascination suggestion, traveler suggestion, etc. In future work, it will attempt to interface all information accessible on various social sites like Facebook, Twitter, and other social sites suggest them just as their companions also.

Pooja Khalokar, et al. [2] Portray the framework takes constant information at that point investigates it, and utilizations the information for ongoing applications to suggest the vacationer's real right intrigued information. The framework gives the decision among three techniques rating suggestion, assessment proposal, and fascination suggestion. It gives bundle gauging to visit organizations and ideas. Later on, it will attempt to interface all information accessible on various social sites like Facebook, Twitter, and other social sites suggest them just as their companions also. Heng-Ru Zhang, et al. [3] In this paper, we have proposed a half-breed recommender framework for the intelligent situation. Through changing the suggested boundaries and contrasting the irregular and half and half calculations, we may reach the accompanying determinations: (1) the proportion of arbitrary proposals has no extraordinary effect on the presentation as long as it isn't too huge (e.g., not more than 0.25), (2) one should utilize $k \mathrm{NN}$ as ahead of schedule as could really be expected, (3) the neighbor's number ought to be sufficiently large (e.g., 45), (4) the review is almost direct increment as for the number of proposals in each round, and (5) the crossover calculation is superior to the arbitrary one.

Cai Chen, et al. [4] In this paper, we proposed another unique user versatile mix technique for crossbreed film suggestion. Contrasting and the customary halfbreed calculation, which utilizes a static blend technique to join the unadulterated calculations, our proposed calculation utilizes a unique procedure that consolidates with the distinctive boundary for various users. Investigations show that our proposed calculation can essentially upgrade the exhibition. Moreover, in this paper, we utilize the outside open asset IMDB as the substantial information on the film, extraordinarily, we utilize the watchwords set as the contribution of the substance-based calculation. Tests show that the catchphrases set gathered from IMDB is a great asset for a suggestion. For future work, considering the dataset accessible is developing so quickly, we will attempt to broaden the versatility of our proposed calculation to suggest the adaptable dataset. We will likewise attempt to take more sorts of assets past the catchphrases set as the contribution of the substance-based algorithm.

Sajal Halder, et al. [5] In this paper, we have shown that users' evaluating-based diversion suggestion framework doesn't give high precision in the proposal cycle. It is similarly imperative to utilize the elements of user conduct after some time. To suggest a thing we have proposed a calculation that utilized the adjustment of the user's inclinations over the long run. At the end of the day, the calculation gives greater need to the as of late evaluated things for proposal. The relationship of time data gives better precision. Simultaneously, we have proposed a mining procedure to prescribe things to the makers with the end goal that they can anticipate users' chose.

Harris Papadakis, et al. [6] Introduced a customized film recommender framework application, created for Android-worked versatile stages. As per our insight, MovieScore is the main portable application in writing customized film proposals. The proposed framework depends on a novel, cutting-edge calculation for customized proposals that shows dynamic gradual update conduct. In this way, when another thing shows up (for example film, user, rating), the calculation is retrained steadily, yielding 
elite and versatile outcomes. The application is not difficult to utilize and gives exact film suggestions to the user on the fly, in light of its customized inclinations. It additionally gives utility functionalities as the development of a "to watch" film rundown and refining the proposal results through separating.

We expect to vigorously enhance the basic recommender calculation, primarily by fusing film metadata in its usefulness. This won't just take into account more precise suggestions yet additionally empower the application to legitimize to the user every proposal provided.

Tianqi Zhou, et al. [7] portray explicit issues of the film proposal, we utilize the Hadoop programming model to execute a suggestion calculation depends on the Item-based CF. Also, we give the acknowledgment of the framework and the comparing exploratory outcomes. The circulated record framework HDFS and conveyed bunch structure MapReduce on the Hadoop stage can store the developing mass of information as well as cycle the information in equal, which improves the presentation of the calculation and the reaction speed of the framework. We accept that the framework that receives the Hadoop procedure can address the issue of huge information and the distributed computing climate.

Jiang Zhang, et al. [8] Fostered a novel cooperative sifting approach called Weighted KM-Slope-VU for quick and adaptable film suggestions, and besides created and conveyed a customized film proposal site, MovieWatch, to furnish users with survey administrations and gather user input on prescribed movie to basically assess our proposed calculation utilizing genuine information. In particular, we received $\mathrm{k}$-intends to parcel users into a few bunches, and afterward for each group imagined a virtual assessment pioneer to address the entirety of the users around there. At that point, rather than handling the first full user thing rating framework, a decreased virtual assessment pioneer thing grid is prepared by the proposed Weighted Slope One-VU suggestion calculation. Analyses of MovieLens datasets show that our plan can accomplish the execution (estimated by RMSE) practically identical with proposal calculations depend on framework factorization, yet diminish time intricacy in like manner situations. Moreover, a common-sense film suggestion framework called MovieWatch was created, conveyed, and opened to the general society to gather user input on the movie pictures prescribed to them. Our plan was then assessed depending on this genuine criticism by enlisting users of MovieWatch.

\section{Propose System}

The proposed framework will contain a data set comprising numerous movies. Continuous investigation guarantees that the framework will adjust progressively dependent on user behaviour. Things are suggested dependent on examinations between thing profiles and user profiles. We can undoubtedly clarify the working of the recommender framework by posting the Content highlights of a thing.

Content-based recommender frameworks use need just the rating of the concerned user and no other user of the framework. It is subject to the connection between users, which suggests that it is content-free.

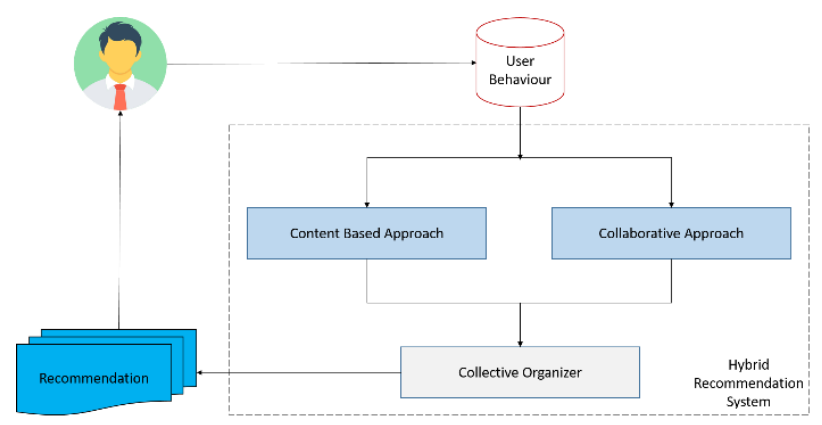

Fig 5: System Architecture 


\section{Flowchart}

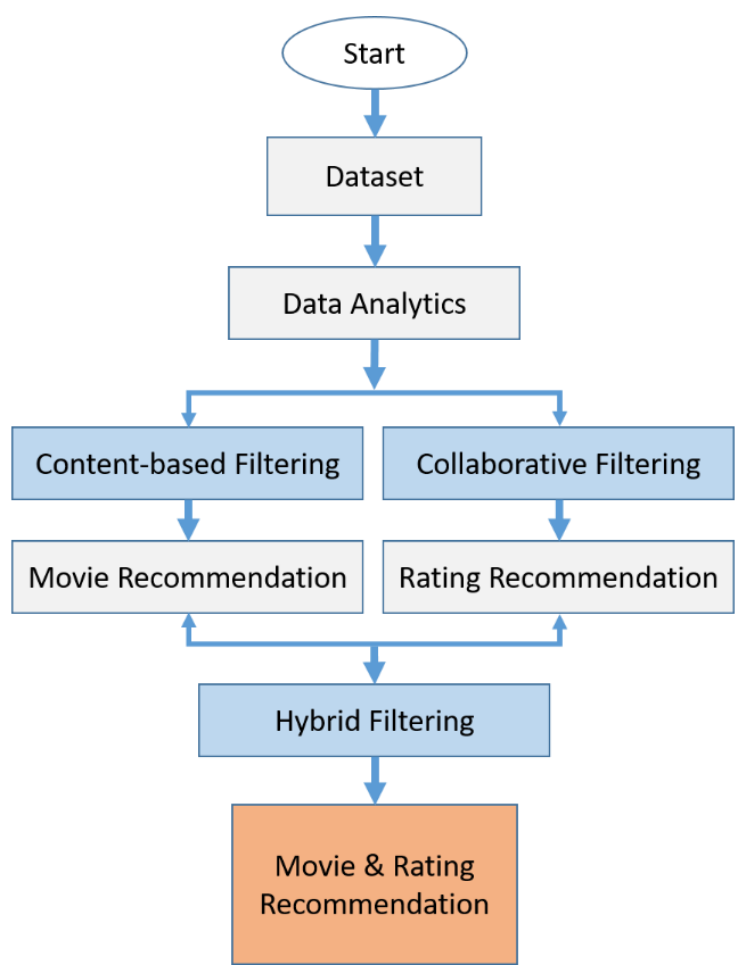

Fig 6: Flowchart

\section{Algorithm}

Gradient descent is applied to the MovieLens informational collection to remove dormant highlights, one of which takes the movie and user predisposition into thought. While looking at the highlights separated from the algorithms there was a solid connection betweens extricated highlights and movie sorts. We show that each element form a particular classification of movie where every movie is addressed as a mix of the classes. It works by circling through each evaluation and movie in the preparation information, attempts to foresee the top max rating and comparable movie.

\section{Input: Training Dataset}

Step 1: Initialize matrices p \& q of size (users $\left.{ }^{*} \mathrm{~K}\right)$ \& $(\mathrm{K}$ * movies with irregular qualities) from a uniform dispersion over $[0,1]$ where $\mathrm{K}$ represents the quantity of highlights that will be removed.

Step 2: Iterate over every one of them noticed ratings in the preparation dataset. (a) Calculate an anticipated rating relating to a genuine rating.

(b) Calculate the mistake for the anticipated rating.

(c) Update $\mathrm{p}$ and $\mathrm{q}$ as indicated by mistake.

Output: Resulset <class_name, similarity_movie, highest_rating>

\section{Dataset}

The Movie dataset contains 1 million user ratings from 6,040 users on 17770 movies. Users of MovieLens were chosen arbitrarily. All users appraised in any event 10 movies. Every user addressed by an exceptional id.

\section{Result}

The best generally results are reached by the thingby-thing-based methodology. It needs 170 seconds to build the model and 3 seconds to anticipate 100,021 ratings. We need a measurement to score or rate a movie. Figure the score for each movie. Sort the scores and prescribe the best-evaluated movie to users.

The best by and large results are reached by the thing- by-thing-based technique. It needs 30 seconds to assemble the model and 3 seconds to expect 10 movies and rating (evaluations). We need an estimation to score or rate a movie. Compute the score for every movie. Sort the scores and endorse the best-assessed movie to users.

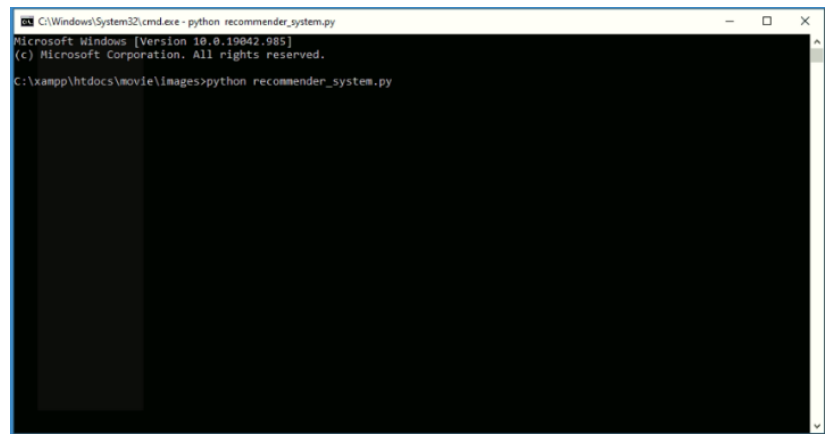

Fig 7: Python Command Prompt (Step 1) 


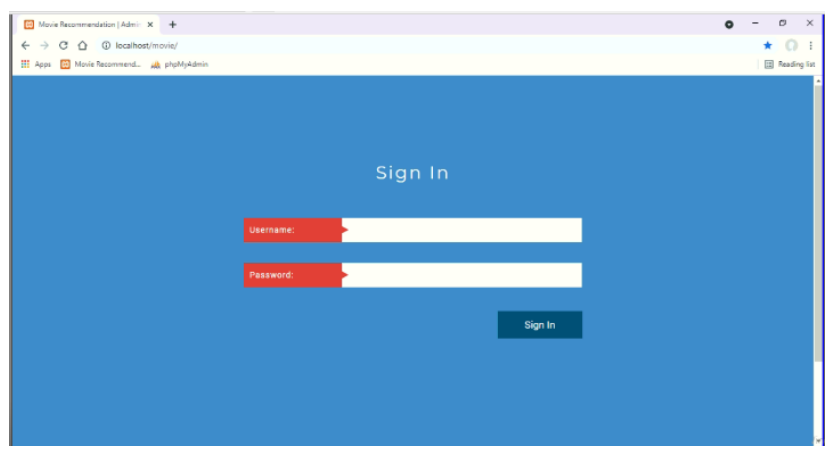

Fig 8: Login Page (Step 2)

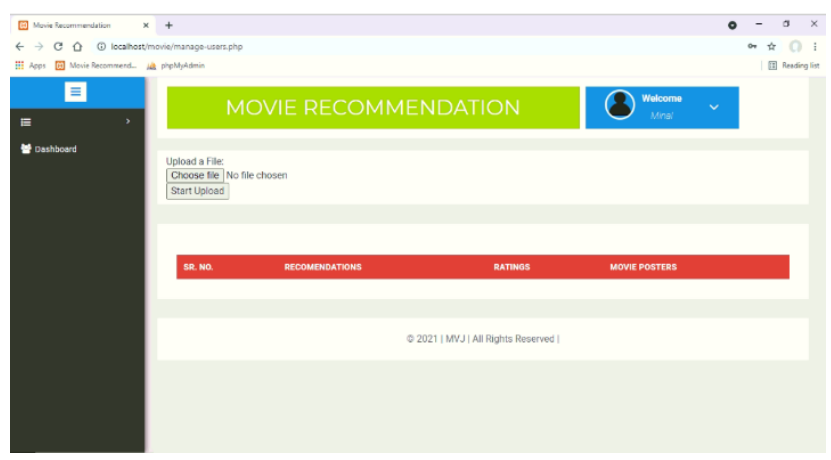

Fig 9: Home Page (Step 3)

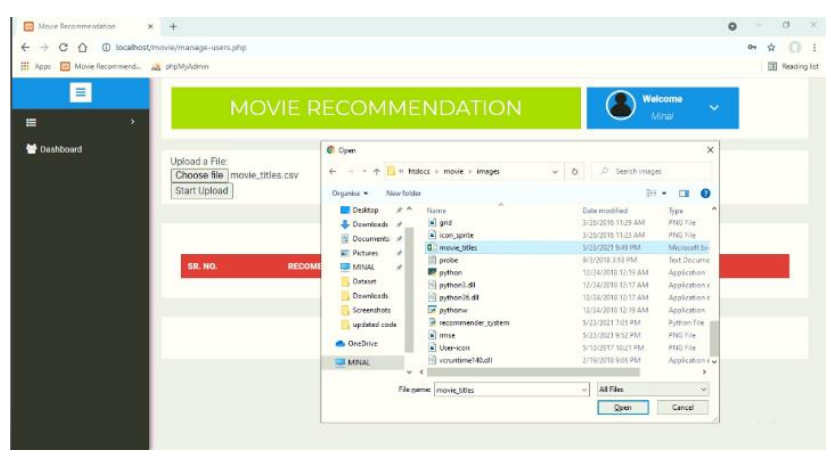

Fig 10: Browse Dataset (Step 4)

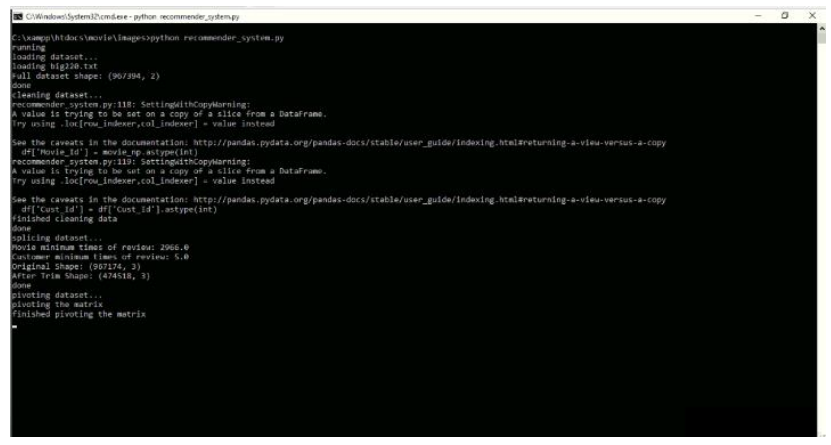

Fig 11: Processing (Step 5)

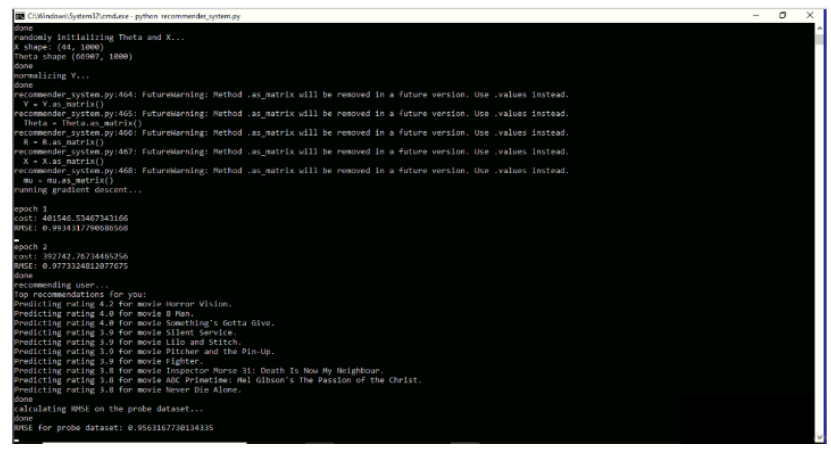

Fig 12: Command Prompt Output - Movie Recommendation (Step 6)

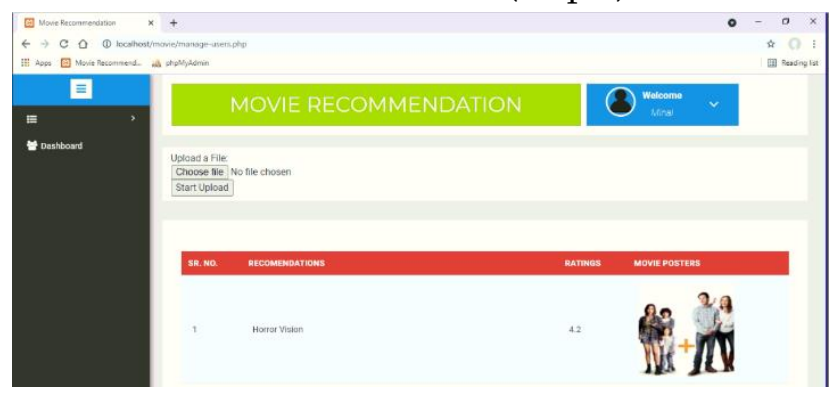

Fig 13: Browser Output - Movie Recommendation (Step 7)

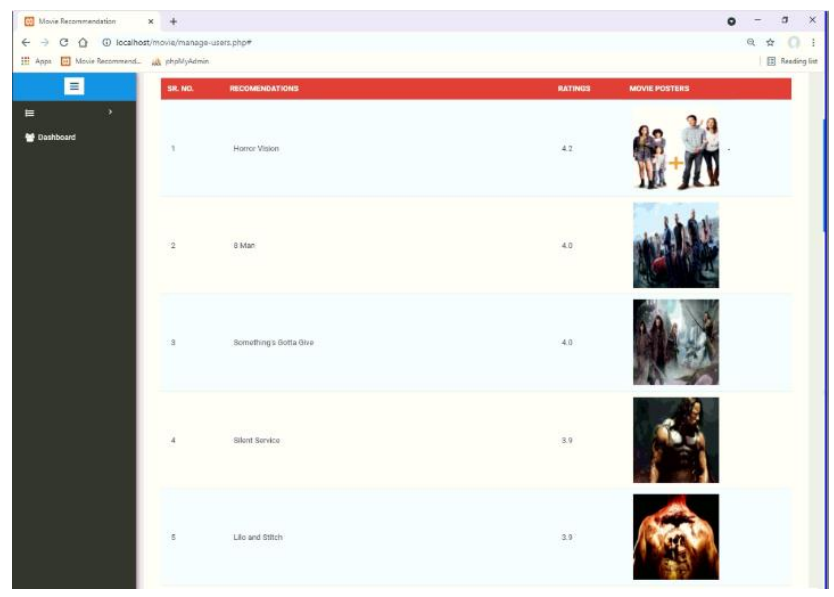

Fig 14: Browser Output - Movie Recommendation 


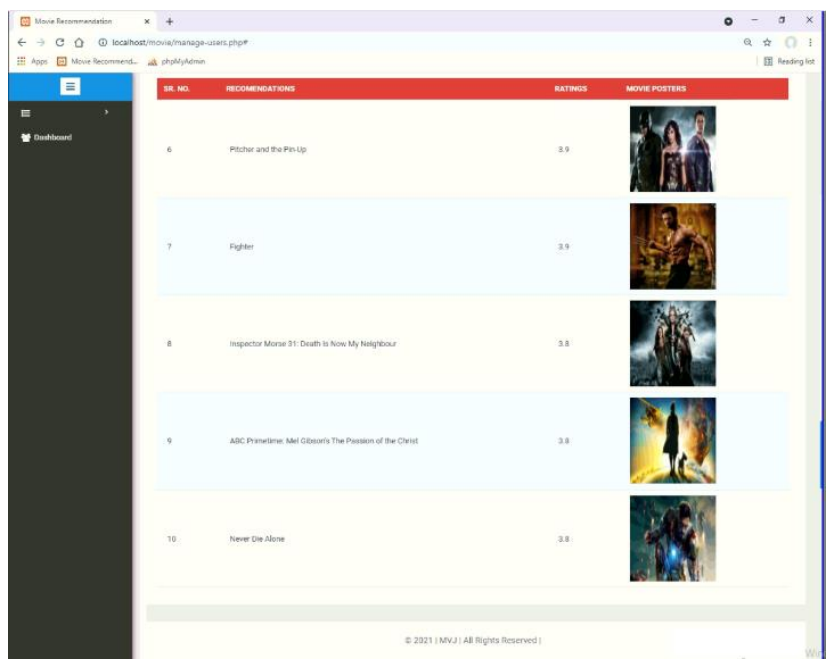

Fig 15: Browser Output - Movie Recommendation

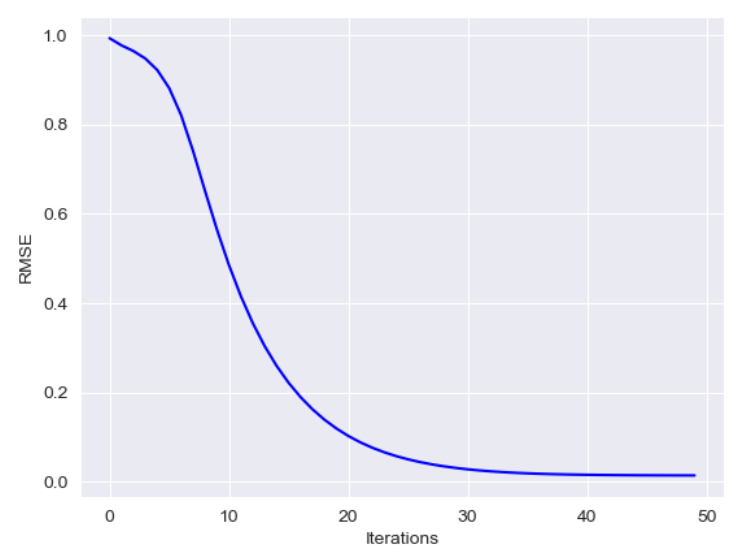

Fig 16: RMSE Graph

\section{Conclusion}

In this paper, we proposed another unique user versatile hybrid technique for movie recommendations. A hybrid recommendations framework that will utilize item-based and user-based separating to give customized suggestions. Notion examination dependent on movie audits and will likewise join a success indicator to appraise the achievement pace of impending movie dependent on different boundaries. The clearest thought is to add highlights to recommend movies with regular entertainers, chiefs, or journalists. The hybrid recommendation approach conquers the downsides of every individual calculation and improves the exhibition of the framework.

\section{VII.Future Scope}

A hybrid recommendation framework can be planned by adding more highlights of information to give a suggestion. Later on, we can deal with a hybrid recommender using grouping and equivalence for better execution. Our approach can be furthermore loosened up to various spaces to propose songs, video, setting, news, books, the travel business, and web business objections, etc.

\section{REFERENCES}

[1]. Pooja P. Khalokar, Prof. Sneha U. Bohra, "Review on Personalized Travel Recommendation According to User Interest using Sentiment Analysis for the Growth of Indian Tourism", International Journal of Research and Analytical Reviews (IJRAR), June 2019.

[2]. Pooja P. Khalokar, Prof. Sneha U. Bohra, "Sentiment Analysis \& Tour Ratings for Best Customized Visit Suggestions for the Expansion of Indian Tourism", International Journal of Research and Analytical Reviews (IJRAR), Sept 2020.

[3]. Heng-Ru Zhang, FanMin, Xu He, and YuanYuan Xu, "A Hybrid Recommender System Based on User-Recommender Interaction", Hindawi Publishing Corporation, Mathematical Problems in Engineering, 2015.

[4]. Cai Chen, Daniel Zeng, "A Dynamic User Adaptive Combination Strategy for Hybrid Movie Recommendation”, IEEE, 2012.

[5]. Sajal Halder, Md. Hanif Seddiqui, and YoungKoo Lee, "An Entertainment Recommendation System using the Dynamics of User Behavior over Time", 17th International Conference on Computer and Information Technology (ICCIT), 2014.

[6]. Harris Papadakis, Paraskevi Fragopoulou, Nikos Michalakis, Costas Panagiotakis, "A Mobile Application for Personalized Movie 
Recommendations with Dynamic Updates", International Conference on Intelligent Systems (IS), 2018.

[7]. Tianqi Zhou, Lina Chen, Jian Shen, "Movie Recommendation System Employing the Userbased CF in Cloud Computing", IEEE International Conference on Computational Science and Engineering (CSE) and IEEE International Conference on Embedded and Ubiquitous Computing (EUC), 2017.

[8]. Jiang Zhang, Yufeng Wang, Zhiyuan Yuan, and Qun Jin, "Personalized Real-Time Movie Recommendation System: Practical Prototype and Evaluation", Tsinghua Science and Technology, 2020.

\section{Cite this article as :}

Minal V. Jamnekar, Prof. Sneha U. Bohra, " A Hybrid Approach for Movie Recommendation based on User Behaviour", International Journal of Scientific Research in Science and Technology(IJSRST), Print ISSN : 2395-6011, Online ISSN : 2395-602X,Volume 8, Issue 3, pp.543-550, May-June-2021. Available at doi $\quad$ : https://doi.org/10.32628/IJSRST2183117 Journal URL : https://ijsrst.com/IJSRST2183117 